\title{
Group seminearrings
}

\author{
Waheed Ahmad Khan ${ }^{\mathrm{a}, *}$, Waqar Arif $^{\mathrm{b}}$, Abdelghani Taouti ${ }^{\mathrm{c}}$ \\ a University of Education, Lahore, Attock Campus, Pakistan. \\ ${ }^{b}$ CIIT Islamabad, Attock campus, Pakistan. \\ ${ }^{c}$ ETS-Maths and NS Engineering Division, HCT University, P. O. Box 7947, Sharjah, United Arab Emirates.
}

\begin{abstract}
In this note, we introduce and discuss the notion of group seminearrings along with few of its characteristics. Firstly, we provide the method of construction of group seminearrings. Then, we introduce different types of ideals of group seminearrings. Finally, we introduce and discuss the group seminearrings homomorphism and amalgamation maps.
\end{abstract}

Keywords: Group seminearrings, ideals, homomorphism.

2020 MSC: 47A15, 46A32, 47D20.

(C)2022 All rights reserved.

\section{Introduction}

Group ring is the amalgamation of two very important and basic algebraic structures, i.e., a finite multiplicative group and a ring. Group rings are first introduced by Aurthur Caylay in 1854. Actually, elements of group ring are in the form of linear combinations of elements of ring and that of group which are involved in the construction of group ring. Elements of group ring has close resemblance with the elements of polynomial rings with single indeterminate. The difference is that if in a polynomial ring we replace the variable along with its powers by the elements of group and replace coefficients with the elements of the ring then they become elements of group ring. Group ring is a unique algebraic structure which is defined and explained in the literature along with its numerous characteristics. Group ring behaves like a ring as its elements satisfying all the axioms of ring but has a unique structure due to its unique construction. Similarly, zero divisors and units of group rings are also very unique. Cohen discussed and explained in detail the zero divisors of group ring [2]. Different types of unit elements of group ring are introduced by Cox et al. in [3]. Structure of group rings and the group of units of integral group rings have been introduced and discussed comprehensively in [8]. Primary group rings have been explored in [1]. Few applications of grouprings towards cryptosystem were explored in [7]. For more details about group rings, we refer to [2-4]. Group nearring is the further generalization of group ring

\footnotetext{
*Corresponding author

Email addresses: sirwakhan2003@yahoo.com \& wakhan@ue.edu.pk (Waheed Ahmad Khan), waqarvicky6699@gmail.com (Waqar Arif), ganitaouti@yahoo.com.au (Abdelghani Taouti)

doi: $10.22436 /$ jmcs.024.04.06
}

Received: 2021-02-14 Revised: 2021-03-10 Accepted: 2021-03-18 
which is introduced and discussed extensively in [10]. Le Riche et al. [10] defined a group nearring in a very special way which is similar to that of matrix nearrings. Group nearring is a subnearring of a nearring $M\left(R^{G}\right)$ which is the set of endomorphisms of a group $R^{G}$. At this point, $R^{G}$ is the direct sum of elements of additive group $(R,+)$ of nearring $R$. Furthermore, number of copies in $R^{G}$ is equal to the order of finite multiplicative group. The elements of group nearring are generated by the function $[r, g]$, where $r \in S, g \in G$, defined by $([r, g](\mu))(h)=r \mu(h g)$ for all $\mu \in S^{G}, h \in G$. Eventually, we define a group seminearring by using the similar approach used for defining group nearring by Le Riche et al. [10]. Different types of ideals of grouprings are discussed in $[5,6]$. For group nearrings one can consult $[5,6,10,11]$.

Group rings have been generalized in terms of group nearrings [10] and group semirings [9]. In this manuscript, we introduce and discuss the notion of group seminearrings along with few of its characterizations. Firstly, we introduce the method of construction of group seminearrings. During this, we follow the method similar to that of group rings and group nearrings. However, we observe that a group seminearring is different structure as compared to that of group rings and group nearrings. Then, we introduce different types of ideals of group seminearring. Finally, we discuss group seminearrings by applying some structure preserving mappings.

\section{Construction of group seminearrings}

In this section, first we provide the method of construction of group seminearring and then we discuss its various basic properties. We begin with the following definition.

Definition 2.1. Let $S$ be a seminearring with identity 1 , and $G$ be a finite multiplicatively written group and $S^{G}$ represents the cartesian direct sum of semigroup $(S,+)$ and number of copies in $S^{G}$ is equal to the order of $G$. Elements of $S^{G}$ are indexed by the elements of group $G$. Let $M\left(S^{G}\right)$ denotes the seminearring of all mappings of a semigroup $S^{G}$ to itself. For some functions in $M\left(S^{G}\right)$, we define the following notations.

(i) The function $\left\langle r, g>\right.$, where $r \in S, g \in G$, defined by $(<r, g>(\mu))(h)=r \mu\left(g^{-1} h\right)$ for all $\mu \in S^{G}$, $h \in G$.

(ii) The function $[r, g]$, where $r \in S, g \in G$, defined by $([r, g](\mu))(h)=r \mu(h g)$ for all $\mu \in S^{G}, h \in G$.

It is notable that the set $\left\{<r, g>: r \in S, g \in G\right.$ \} generates a sub-seminearring of $M\left(S^{G}\right)$ and we denote it by $S<G>$. Similarly the collection $\{[r, g]: r \in S, g \in G\}$ generates a sub-seminearring of $M\left(S^{G}\right)$ and will be denoted as $S[G]$. Actually these sub-seminearrings are our group seminearrings. However, if we replace (i) of Definition 2.1 with $\left(i^{\prime}\right)$ the function $\langle r, g\rangle$, where $r \in S, g \in G$, defined by $(<r, g\rangle$ $(\mu))(h)=r \mu(g h)$ for all $\mu \in S^{G}, h \in G$, then, we can easily conclude that: if $G$ is an abelian group then we have $S<G>=S[G]$. On the other hand, we show that if $G$ is not an abelian group then there does exist a natural isomorphism between two sub-nearsemirings $S<G>$ and $S[G]$ of $M\left(S^{G}\right)$.

Theorem 2.2. Group seminearrings $\mathrm{S}<\mathrm{G}>$ and $\mathrm{S}[\mathrm{G}]$ of $\mathrm{M}\left(\mathrm{S}^{\mathrm{G}}\right)$ are same if group $\mathrm{G}$ is abelian otherwise they are isomorphic to each other, i.e., $\mathrm{S}<\mathrm{G}>\cong \mathrm{S}[\mathrm{G}]$.

Proof. Let $G$ be an abelian group w.r.t multiplication, then for all $\mu \in S^{G}, h \in G$, we have $(<r, g>$ $(\mu))(h)=r \mu(g h)=r \mu(h g)=([r, g](\mu))(h)$. This implies that both $S<G>$ and $S[G]$ are equal. On the other hand, if we consider $\mathrm{G}$ a nonabelian multiplicative group then we show that there is a natural isomorphism between $S<G>$ and $S[G]$. For this, let $\phi: R^{G} \rightarrow R^{G}$ be a function defined by $(\phi(\mu))\left(h_{1}\right)=$ $(\phi(\mu))\left(g_{1} g_{2}\right)=\mu\left(g_{2} g_{1}\right)=\mu\left(h_{2}\right)$, for all $\mu \in S^{G}$ and $h_{1}, h_{2}, g_{1}, g_{2} \in G$. Then, it can be easily shown that $\phi \in \operatorname{Aut}\left(R^{\mathrm{G}}\right)$. It is notable that $\phi^{2}=e$ which implies $\phi^{-1}=\phi$. We have to show that the automorphism say $\phi^{*}$ of $M\left(S^{G}\right)$ maps $S<G>$ onto $S[G]$. Consider $\phi^{*}(<r, g>)=\phi(r, g) \phi^{-1}=\phi<r, g>\phi$. For all $\mu \in S^{G}, h_{1} \in G$, we have $((\phi<r, g>\phi) \mu)\left(h_{1}\right)=((\phi<r, g>\phi) \mu)\left(g_{1} g_{2}\right)=(\phi<r, g>) \mu\left(g_{2} g_{1}\right)=(\phi<$ $r, g>) \mu\left(h_{2}\right)=\phi\left(r \mu\left(h_{2} g\right)\right)=r \phi\left(\mu\left(h_{2} g\right)\right)=\left(r \mu\left(g h_{2}\right)=([r, g](\mu))\left(h_{2}\right)\right.$. Hence $\phi^{*}(<r, g>)=[r, g]$ for all $r \in S, g \in G$. Hence, this implies that $\phi^{*}(S<G>)=S[G]$. 
On the other hand, if we keep carry on with Definition 2.1, then we can deduce the below result which is similar to that for group nearrings.

Theorem 2.3. Group seminearrings $\mathrm{S}<\mathrm{G}>$ and $\mathrm{S}[\mathrm{G}]$ of $\mathrm{M}\left(\mathrm{S}^{\mathrm{G}}\right)$ are isomorphic to each other.

Proof. Similar to that of group nearring [10, Theorem 2.2].

Let $S[G]$ be a group seminearring constructed from a seminearring $S$ and a finite multiplicatively written group $G$. As $S$ is always a subring of a group seminearring it means if $S$ is a left seminearring then $S[G]$ will be the left group seminearring. Similarly, if $S$ is right seminearring then $S[G]$ is right group seminearring.

Corollary 2.4. Let $\mathrm{m}$ be the order of a group seminearring $\mathrm{S}$ and $\mathrm{n}$ be the order of a group $\mathrm{G}$. Then, the group seminearring $\mathrm{S}[\mathrm{G}]$ is of order $|\mathrm{R}|^{|\mathrm{G}|}=\mathrm{m}^{\mathrm{n}}$.

Example 2.5. Let $S=\{0, a, b, c, d\}$ be a right nearsemiring with the operations tables given below.

\begin{tabular}{|c|c|c|c|c|c|c|c|c|c|c|c|}
\hline+ & 0 & $a$ & $\mathrm{~b}$ & $c$ & $d$ & & 0 & $a$ & $b$ & $c$ & $\mathrm{~d}$ \\
\hline 0 & 0 & $a$ & $b$ & $\mathrm{c}$ & $\mathrm{d}$ & 0 & 0 & 0 & 0 & 0 & 0 \\
\hline$a$ & $a$ & $a$ & $b$ & $d$ & $d$ & $a$ & 0 & $a$ & $b$ & $\mathrm{~b}$ & $d$ \\
\hline$b$ & $b$ & $b$ & $b$ & $\mathrm{~d}$ & $d$ & $b$ & 0 & $a$ & $b$ & $\mathrm{~b}$ & $\mathrm{~d}$ \\
\hline$c$ & $c$ & $\mathrm{~d}$ & $\bar{d}$ & $\mathrm{c}$ & $\mathrm{d}$ & $c$ & 0 & $a$ & $\mathrm{~b}$ & $\mathrm{~b}$ & $\mathrm{~d}$ \\
\hline $\mathrm{d}$ & $\mathrm{d}$ & $d$ & $\mathrm{~d}$ & $\mathrm{~d}$ & $\mathrm{~d}$ & $\mathrm{~d}$ & 0 & $a$ & $b$ & $\mathrm{~b}$ & $d$ \\
\hline
\end{tabular}

Let $R=\{0, a, b\}$ be a sub-seminearring of $S$ and we will construct here a group seminearring using seminearring $R$ and group $G=C_{2}=\left\{x: x^{2}=e\right\}$. As $R^{G}$ represents the cartesian direct sum of order of $G$ copies of $(R,+)$ so here we have $R^{G}=\{(0,0)(0, a)(0, b)(a, 0)(a, a)(a, b)(b, 0)(b, a)(b, b)\}$. We construct our group seminearring by function $([r, g](\mu))(h)=r \mu(h g)$ for all $\mu \in R^{G}, h \in G$. For understanding, we check it, i.e., for any $\mu=(0, a)$ and $h=0$, we have $([b, x](0, a))(e)=b(0, a)(e x)=(b .0, b . a)(x)=$ $(0, a) x=\{0 e+a x\} x=0(e . x)+a(x . x)=0 x+a e=a e+0 x \in R[G]$. Clearly, we have 9 choices for $\mu$. Hence, by the above corollary, the order of group seminearring is $|\mathrm{R}|^{|\mathrm{G}|}=3^{2}=9$.

Lemma 2.6. Let $\mathrm{S}[\mathrm{G}]$ be the group seminearring. Let $\mathrm{r}, \mathrm{s} \in \mathrm{S}, \mathrm{g}_{1}, \mathrm{~g}_{2} \in \mathrm{G}$, then following holds for every value of the parameters.

(1) $\left[r, g_{1}\right]\left[s, g_{2}\right]=\left[r s, g_{1} g_{2}\right]$;

(2) $[r, g]+[s, g]=[r+s, g]$.

Proof. Let $\mu \in S^{G}$ and $h \in G$. Then by the definition of a group seminearring, we have

(1) $\left(\left(\left[r, g_{1}\right]\left[s, g_{2}\right]\right)(\mu)\right)(h)=\left(\left[r, g_{l}\right]\left(\left[s, g_{2}\right](\mu)\right)\right)(h)=r\left(\left[s, g_{2}\right](\mu)\right)\left(h g_{l}\right)=r s\left(\mu\left(h g_{1} g_{2}\right)\right)=\left(\left[r s, g_{l} g_{2}\right](\mu)\right)(h)$.

(2) Similarly, $(([r, g]+[s, g])(\mu))(h)=[r, g](\mu)(h)+[s, g](\mu)(h)=r \mu(h g)+s \mu(h g)=(r+s) \mu(h g)=[r+$ $s, g](\mu)(h)$.

The above lemma is also valid for $\langle r, g\rangle$, where $r \in S$ and $g \in G$.

Lemma 2.7. Let $\mathrm{S}[\mathrm{G}]$ be group seminearring, $\mathrm{R}[\mathrm{G}]$ a group nearring, and $\mathrm{RG}$ a group ring, then:

(i) $\mathrm{S}[\mathrm{G}] \cong \mathrm{R}[\mathrm{G}]$, if seminearring $\mathrm{S}$ is replaced with nearring $\mathrm{R}$;

(ii) $S[G] \cong R G$, if seminerring $S$ is replaced with standard ring $R$.

Now we introduce few more terminologies regarding the elements of group seminearring, i.e., generating sequence, complexity and support of the elements.

Definition 2.8. Let $S[G]$ be a group seminearring and $\alpha \in S[G]$ and there exist a finite sequence of elements of $S[G]$, i.e., $\alpha_{1}, \alpha_{2}, \ldots, \alpha_{n}$, such that $\alpha_{i}=\alpha$, for all $1 \leqslant i \leqslant n$. This finite sequence is called generating sequence for the element $\alpha$. 
Remark 2.9. One of the below conditions must be satisfied for a generating sequence of an element $\alpha \in$ $\mathrm{S}[\mathrm{G}]$.

(i) $\alpha_{i}=[s, g]$ for some $s \in S, g \in G$.

(ii) $\alpha_{i}=\alpha_{k}+\alpha_{l}$ for some $k$ and $l$, where $1 \leqslant k, l<i$.

(iii) $\alpha_{i}=\alpha_{k} \alpha_{l}$ for some $k$ and $l$, where $1 \leqslant k, l<i$.

Definition 2.10. Complexity of an element of a group seminearring is the length of generating sequence of minimum length for that element. The complexity for any $\alpha \in S[G]$ is denoted by $c(\alpha)$.

Remark 2.11. Complexity of an element can be either equal to 1 or greater then 1, i.e., for all $\alpha \in \mathrm{S}[\mathrm{G}]$ we have always $c(\alpha) \geqslant 1$. We have $c(\alpha)=1$ iff $\alpha=[r, g]$ for some $r \in S, g \in G$. Moreover, for $c(\alpha)>1$, we have either $\alpha_{i}=\alpha_{k}+\alpha_{l}$ or $\alpha_{i}=\alpha_{k} . \alpha_{l}$ with $c\left(\alpha_{k}\right), c\left(\alpha_{l}\right)<c\left(\alpha_{i}\right)$.

Definition 2.12. The support of any element of $S^{G}$ say $\mu \in S^{G}$, is denoted by $\operatorname{supp}(\mu)$, and is defined as: $\operatorname{supp}(\mu):=\{x \in G \mid \mu(x) \neq 0\}$. $S^{(G)}$ is the collection of elements of $S^{G}$ which has finite support and $S^{(G)}$ is the subgroup of $S^{G}$.

Theorem 2.13. $\mathrm{S}^{(\mathrm{G})}$ is a faithful $\mathrm{S}[\mathrm{G}]$-module.

Proof. It is immediate from [10, Theorem 2.5 ].

\section{Ideals of group seminearrings}

In this section we provide the theory of ideals of group seminearrings.

Definition 3.1. Let $S$ be a seminearring. A subset $L \subseteq S$ is said to be an ideal of $S$, if either $L S \subseteq L$ or $\mathrm{SL} \subseteq \mathrm{L}$.

Here we will introduce and discuss different types of ideals of group seminearring $S[G]$. Let $S[G]$ be a group seminearring constructed from a seminearring $S$ and a finite multiplicatively written group $G$. As we have discussed earlier that seminearring $S$ is always a subring of a group seminearring $S[G]$, so we may explore the ideals of group seminearrings. We denote the ideal of any group seminearring $S[G]$ by I[G] or simply IG.

Theorem 3.2. Let $\mathrm{I} \subseteq \mathrm{S}$ be an ideal of seminearring $\mathrm{S}$. Then $\mathrm{IG} \subseteq \mathrm{S}[\mathrm{G}]$ is an ideal of a group seminearring, where $\mathrm{IG}=\left\{\Sigma_{\mathrm{g} \in \mathrm{G}} \mathrm{r}_{\mathrm{g}} \mathrm{h} \mid \mathrm{r}_{\mathrm{g}} \in \mathrm{I}, \mathrm{h} \in \mathrm{G}\right\}$.

Proof. It is clear that the subset IG of a group seminearring is closed with respect to the addition operation. Further to this, let $\alpha=\Sigma_{g \in G} r_{g} g \in S[G]$ and $\beta=\Sigma_{g \in G} i_{g} h \in I G$. Then we have $\alpha \beta=$ $\left(\Sigma_{g \in G} r_{g} g\right)\left(\Sigma_{g \in G} i_{g} h\right)=\sum_{g \in G}\left(r_{g} i_{g}\right)(g h)$. Since I is an ideal of a seminearring $S$, we have $r_{g} i_{g}=j_{g} \in I$, and since $\mathrm{G}$ is a multiplicative group so $\mathrm{gh}=\mathrm{k} \in \mathrm{G}$. Hence, we have $\alpha \beta=\Sigma_{g \in G} j_{g} k \in$ IG. Which implies that IG is an ideal of a group seminearring $S G$.

Corollary 3.3. Let $\mathrm{IG} \subseteq \mathrm{SG}$ be an ideal of a group seminearring, then $((\mathrm{SG}) /(\mathrm{IG})) \cong((\mathrm{S} / \mathrm{I})) \mathrm{G}$.

Definition 3.4. Let IG be an ideal of a group seminearring S[G]. Then IG is said to be a prime ideal if one of the following holds:

(i) if $\left[r_{1}, g\right]\left[r_{2}, h\right] \in I G$, then either $\left[r_{1}, g\right] \in I G$ or $\left[r_{2}, h\right] \in I G$, where $r_{1}, r_{2} \in R$ and $g$, $h \in G$;

(ii) for any two ideals $J G$ and $K G$ of a $S[G]$ if $(J G)(K G) \subseteq I G$, then either $J G \subseteq I G$ or $K G \subseteq I G$.

Definition 3.5. Let IG be an ideal of a group seminearring $S[G]$. Then IG is said to be a weakly prime ideal if for any two ideals JG and $K G$ of a $S[G]$, if $0 \neq(J G)(K G) \subseteq$ IG then either $J G \subseteq I G$ or $K G \subseteq$ IG.

Theorem 3.6. Let IG be a weakly prime ideal of a group seminearring $\mathrm{S}[\mathrm{G}]$ which is not prime. Then $(\mathrm{IG})^{2}=0$. 
Proof. Let $(\mathrm{IG})^{2} \neq 0$, we will prove that IG is a prime ideal. Let for any $\alpha, \beta \in \mathrm{S}[\mathrm{G}]$, we have $\alpha \beta \in$ IG. If $0 \neq \alpha \beta$ then by the definition of weakly prime ideal we have either $\alpha \in$ IG or $\beta \in$ IG. We assume that $\alpha \beta=0$. Let $\alpha(\mathrm{IG}) \neq 0$. For any $v=\Sigma_{g \in G} i_{g} h \in$ IG say $\alpha v=\left(\Sigma_{g \in G} r_{g} g\right)\left(\Sigma_{g \in G} i_{g} h\right)=\Sigma_{g \in G}\left(r_{g} i_{g}\right) g h=$ $\Sigma_{\mathrm{g} \in \mathrm{G}} \mathrm{s}_{\mathrm{g}} \mathrm{k} \neq 0$. Then, we have $0 \neq \alpha v=\alpha(\beta+\nu) \in$ IG which implies that either $\alpha \in$ IG or $(\beta+v) \in$ IG. Hence, $\alpha \in$ IG or $\beta \in$ IG. So, we assume that $\alpha($ IG $)=0$ and similarly we can assume that $\beta($ IG $)=0$. As $(\mathrm{IG})^{2}=0$ so there exist $\nu, \eta \in$ IG with $v \eta \neq 0$. Then, we have $0 \neq v \eta=(\alpha+\nu)(\beta+\eta) \in$ IG, so this implies that $\alpha+\nu \in$ IG or $\beta+\eta \in$ IG, which implies $\alpha \in$ IG or $\beta \in$ IG. Hence IG is a prime ideal.

For any ideal I of seminearring $S$ there exist some ideals of the group seminearring $S[G]$ denoted by $\mathrm{I}^{+}$and $\mathrm{I}^{*}$ and can be constructed from the ideal $\mathrm{I}$ of a seminearring $S$ which is directly involved in the construction of SG.

Definition 3.7. Let $S[G]$ be a group seminearring constructed from a seminearring $S$ and a finite multiplicatively written group $\mathrm{G}$. Let $\mathrm{I} \subseteq \mathrm{S}$ be an ideal of $S$, then:

(i) $\mathrm{I}^{+}$is an ideal in $\mathrm{S}[\mathrm{G}]$, where $\mathrm{I}^{+}=\mathrm{Id}<[i, e]: i \in \mathrm{I}>$;

(ii) $\mathrm{I}^{*}$ is an ideal in $\mathrm{S}[\mathrm{G}]$, where $\mathrm{I}^{*}=\left(\mathrm{I}^{\mathrm{G}}: \mathrm{S}^{\mathrm{G}}\right)=v \in \mathrm{S}[\mathrm{G}] \mid \nu\left(\mathrm{S}^{\mathrm{G}}\right) \subseteq \mathrm{I}^{\mathrm{G}}$.

For the two ideals defined above the ideal $\mathrm{I}^{+} \subseteq \mathrm{I}^{*}$.

Theorem 3.8. Let $\mathrm{I}$ be any ideal of a seminearring $\mathrm{S}$. If $\mathrm{I}^{+}$is nilpotent in $\mathrm{S}[\mathrm{G}]$, then $\mathrm{I}$ is nilpotent in $\mathrm{S}$.

Proof. Let $\mathrm{I}^{+}$be a nilpotent in $S[G]$, then for any $k \in N$, we have $\left(I^{+}\right)^{k}=0$. For any $i_{1}, i_{2}, i_{3}, \ldots, i_{k} \in I$, we have $\left[i_{1}, e\right] .\left[i_{2}, e\right] .\left[i_{3}, e\right] \ldots,\left[i_{k}, e\right]=\left[i_{1} i_{2} i_{3} \ldots, i_{k}, e\right]=0$. This implies that $\left(\left[i_{1} i_{2} i_{3} \ldots, i_{k}, e\right] \mu\right) h=0 \Rightarrow$ $\left(i_{1} i_{2} i_{3} \ldots, i_{k}\right) \mu(h e)=0 \Rightarrow\left(i_{1} i_{2} i_{3} \ldots, i_{k}\right) \mu(h)=0$, for all $\mu \in R^{G}$ and $h \in G$. Particularly, for the function $\mu(h)=1$, we have $i_{1} i_{2} i_{3} \ldots, i_{k}=0$. Hence, we have proved that $I$ is a nilpotent ideal in $S$.

Lemma 3.9. For any ideals $\mathrm{I}, \mathrm{J}$ and $\mathrm{K}$ of a seminearring $\mathrm{S}$, if $\mathrm{JK} \subseteq \mathrm{I}$, then $\mathrm{J}^{+} \mathrm{K}^{*} \subseteq \mathrm{I}^{*}$.

Theorem 3.10. Let $\mathrm{S}$ be a seminearring and $\mathrm{I}$ be any ideal of $\mathrm{S}$ and if $\mathrm{I}^{+}$is prime in $\mathrm{S}[\mathrm{G}]$, then so is $\mathrm{I}$ in $\mathrm{S}$.

Proof. Let I be an ideal of seminearring $\mathrm{S}$ and $\mathrm{I}^{+}$be an ideal of $\mathrm{S}[\mathrm{G}]$. Let $\mathrm{I}^{+}$be a prime ideal and also let $\mathrm{J}$ and $\mathrm{K}$ be any two ideals of $\mathrm{S}$ such that $\mathrm{J}, \mathrm{K} \nsubseteq \mathrm{I}$. Since $\mathrm{J} \nsubseteq \mathrm{I} \Rightarrow \mathrm{J}^{+} \nsubseteq \mathrm{I}$ and $\mathrm{K} \nsubseteq \mathrm{I} \Rightarrow \mathrm{K}^{*} \nsubseteq \mathrm{I}$. This implies that $\mathrm{J}^{+} \mathrm{K}^{*} \nsubseteq \mathrm{I}$. Since an ideal $\mathrm{I}^{+}$is prime in $\mathrm{S}[\mathrm{G}]$ and $\mathrm{J}^{+} \mathrm{K}^{*} \nsubseteq \mathrm{I}$, it means JK $\nsubseteq \mathrm{I}$ (by the above lemma). Hence $I$ is a prime ideal of $S$.

Corollary 3.11. Let $\mathrm{S}[\mathrm{G}]$ be a group seminearring constructed from seminerring $\mathrm{S}$ and a finite multiplicatively written group $\mathrm{G}$. For any ideal $\mathrm{I}$ of $\mathrm{S}$, the maps $\mathrm{I} \rightarrow \mathrm{I}^{+}$and $\mathrm{I} \rightarrow \mathrm{I}^{*}$ are injective.

Theorem 3.12. Let $\lambda: \mathrm{R}[\mathrm{G}] \rightarrow \mathrm{S}[\mathrm{G}]$ be a group seminearring homomorphism. If IG is a prime ideal of $\mathrm{S}[\mathrm{G}]$, then $\lambda^{-1}(\mathrm{IG})$ is a prime ideal of $\mathrm{R}[\mathrm{G}]$.

Proof. Let $\alpha \beta \in \lambda^{-1}(\mathrm{IG})$ it means $\lambda(\alpha \beta) \in \mathrm{IG} \Rightarrow \lambda(\alpha) \in$ IG or $\lambda(\beta) \in$ IG. As IG is a prime ideal of $S[G]$ which implies $\alpha \in \lambda^{-1}$ (IG) or $\beta \in \lambda^{-1}$ (IG). Thus $\lambda^{-1}$ (IG) is a prime ideal of $R[G]$.

\section{Group seminearring homomorphism}

Let $R$ and $S$ be two seminearrings and $\eta$ be an epimorphism between them say $\eta: R \rightarrow S$. Let $G$ be finite multiplicatively written group then clearly $R^{G}$ and $S^{G}$ denote the cartesian direct sum of $(R,+)$ and $(S,+)$ of order of $G$ copies, respectively. Let $\omega: R^{G} \rightarrow S^{G}$ be an epimorphism defined by $\omega(\mu)(g)=\omega((\mu(g))$ for all $g \in G$. For seminearrings $R$ and $S$ and finite multiplicative group $G, R[G]$ and $S[G]$ are two corresponding group seminearrings, respectively.

Lemma 4.1. Let $\omega: R^{G} \rightarrow S^{G}$ be an epimorphism and let for any $u, v \in R^{G}$ if $\omega(u)=\omega(v)$, then for all $M \in R[G], \omega(M u)=\omega(M v)$. 
Proof. We use the concept of induction to prove this lemma. Let $A=B C$, then clearly we have $c(B), c(C)<$ $c(A)$. Let $\omega^{-1}: S^{G} \rightarrow R^{G}$ be the inverse mapping of $\omega$, such that for any $\beta \in S^{G}, \omega^{-1} \omega(\beta)=\beta$. Then clearly for all $\mu \in R^{G}$, we have $\omega(\mu)=\omega\left(\omega \omega^{-1}(\mu)\right)$. So, by using induction hypothesis for all $\mu \in R^{G}$, we have $\omega(\beta \mu)=\omega\left(\beta \omega \omega^{-1}(\mu)\right)$. As $\omega(u)=\omega(v)$, so $\omega(A \mu)=\omega(B C \mu)=\omega\left(B \omega \omega^{-1}(C \nu)\right)=\omega(B C v)=$ $\omega(A v)$, hence proved.

We can easily define an epimorphism between two group seminearrings, let $R[G]$ and $S[G]$ be the two group seminearrings, then $\lambda: R[G] \rightarrow S[G]$ defined by $\lambda(A)(\beta)=\omega\left(A \omega^{-1}(\beta)\right)$, where $\beta \in S^{G}$, is a group seminearring epimorphism.

Theorem 4.2. For any two seminearrings $\mathrm{R}$ and $\mathrm{S}$ if $\eta: \mathrm{R} \rightarrow \mathrm{S}$ is an epimorphism, then $\lambda: \mathrm{R}[\mathrm{G}] \rightarrow \mathrm{S}[\mathrm{G}]$ is also a ring homomorphism of group seminearrings.

Proof. Let $\lambda: R[G] \rightarrow S[G]$ is defined by $\lambda(A)(\beta)=\omega\left(A \omega^{-1}(\beta)\right)$, where $\beta \in S^{G}$. So, for any $L, M \in R[G]$ we have,

(i) $\lambda(M+N)(\beta)=\omega\left((M+N) \omega^{-1}(\beta)\right)=\omega\left(\left(M \omega^{-1}(\beta)+N \omega^{-1}(\beta)\right)=\omega\left(\left(M \omega^{-1}(\beta)\right)+\omega\left(N \omega^{-1}(\beta)\right)=\right.\right.$ $\lambda(M)(\beta)+\lambda(N)(\beta)$

(ii) $\lambda(M N)(\beta)=\omega\left((M N) \omega^{-1}(\beta)\right)=\omega\left(\left(M \omega^{-1} \omega N\left(\omega^{-1} \beta\right)=\lambda(M)\left(\omega N \omega^{-1}(\beta)\right)=\lambda(M) \lambda(N)(\beta)\right.\right.$.

The group seminearring homomorphism presented in the above theorem is an epimorphism.

Remark 4.3. If ker $\eta=\psi$, then for group seminearring epimorphism, i.e., $\lambda: R[G] \rightarrow S[G]$, we have $\operatorname{ker} \lambda=\psi^{*}=\left(\psi^{\mathrm{G}}: \mathrm{R}^{\mathrm{G}}\right)=\left\{A \in \mathrm{R}^{\mathrm{G}} \mid A \mu \subseteq \psi^{\mathrm{G}}\right.$ for all $\left.\mu \in \mathrm{R}^{\mathrm{G}}\right\}$.

Note that if $\operatorname{ker} \eta=\psi$ is any ideal of $R$, then $\operatorname{ker} \lambda=\psi^{*}$ will be the ideal of group seminearring $R[G]$.

Augmentation map has been defined and discussed in the context of group rings and group nearrings, we initiate the notion of augmentation map for group seminearrings. It is actually a map from a group seminearring to a seminearring which is involved in the construction of a group seminearring.

Definition 4.4. Let $S[G]$ be a group seminearring, then $\gamma: S[G] \rightarrow S$ defined by $\gamma([r, g])=\gamma\left(\Sigma_{g \in G} s_{g} g\right)=$ $\Sigma_{g \in G} s_{g}=[r, 1]$ is called augmentation map for group seminearring.

The kernel of augmentation map consists of all those elements of $S[G]$ say a, where $a=\Sigma_{g \in G} r_{g} g$ such that $\gamma(a)=\gamma\left(\Sigma_{g \in G} r_{g} g\right)=0$. The kernel of augmentation map is an ideal and it is called augmentation ideal and it is represented by $\triangle$.

Lemma 4.5. Let $\mathrm{S}[\mathrm{G}]$ be a group seminearring constructed from a seminearring $\mathrm{S}$ and a finite multiplicatively written group $\mathrm{G}$. For an augmentation map $\gamma$, if $\triangle$ is the augmentation ideal of $\mathrm{S}[\mathrm{G}]$, then we have $((\mathrm{S}[\mathrm{G}]) / \Delta) \cong \mathrm{S}$.

Lemma 4.6. The augmentation ideal in group seminearring $\mathrm{S}[\mathrm{G}]$ is generated by the element $\{[1, \mathrm{~g}]-[1, \mathrm{e}] \mid \mathrm{g} \in \mathrm{G}\}$.

\section{Conclusion}

In this article, we have introduced and discussed the notion of group seminearrings and provided few of its characteristics. We have provided the method of construction of group seminearrings and different types of ideals of group rings are also explored. At the end we have introduced the concepts of group seminearrings homomorphism and amalgamation maps. At the base of the results obtained in this note, one can discuss the concepts related to group rings in the setting of group seminearrings.

\section{Acknowledgement}

This manuscript containing some results which are extracted from the MS thesis of the second author (Waqar Arif). 


\section{References}

[1] A. Y. M. Chin, K. T. Qua, Primary group rings, Rend. Semin. Mat. Univ. Padova, 137 (2017), 223-228. 1

[2] J. M. Cohen, Zero divisors in group ring, Comm. Algebra, 2 (1974), 1-14. 1

[3] D. Cox, Units in Group Rings, Lakehead University, Thunder Bay, Ontario, (2016). 1

[4] D. S. Dummit, R. M. Foote, Abstract Algebra, third edition, John Wiley \& Sons, Hoboken, (2004). 1

[5] R. L. Fray, On Ideals in Group Near-rings, Acta Math. Hungar., 74 (1997), 155-165. 1

[6] N. J. Groenewald, S. Juglal, J. H. Meyer, Prime ideals in group near-rings, Algebra Colloq., 15 (2008), 501-510. 1

[7] S. Inam, R. Ali, A new ElGamal-like cryptosystem based on matrices over groupring, Neural Comput. Appl., 29 (2018), 1279-1283. 1

[8] E. Jespers, Structure of group rings and the group of units of integral group rings: an invitation, arXiv preprint, (2020), 39 pages. 1

[9] W. B. V. Kandasamy, Smarandache semirings, semifields and semivector spaces, American Research Press, Rehoboth, (2002). 1

[10] L. R. Le Riche, J. D. P. Meldrum, A. P. J. van der Walt, On Group Near-rings, Arch. Math., 52 (1989), 132-139. 1, 2, 2

[11] J. D. P. Meldrum, J. H. Meyer, The augmentation ideal in group near-rings, Monatsh. Math., 156 (2009), $313-323.1$ 